\title{
THE PZC OF MERCURY IN THE PRESENCE OF HUMIC ACIDS AND THEIR COMPLEXES WITH ALUMINIUM
}

\author{
GERALDINE S.P. RITCHIE * and ALAN M. POSNER \\ Department of Soul Science and Plant Nutrition, University of Western Australia, Nedlands, \\ Western Australia, 6009 (Australia)
}

\section{IAN M RITCHIE}

Department of Physical and Inorganic Chemustry, University of Western Australia, Nedlands, Western Australıa, 6009 (Australia)

\begin{abstract}
Variation of the lifetime of a mercury drop with potential was used to determine the pzc of mercury in the presence of soil humic acids and their aluminium complexes In all cases there was an overall net shift in the pzc in the cathodic direction. Varıation in the extent of the shift with $\mathrm{pH}$ and concentration indicated greater adsorption of negatively charged species The shift was smaller in the presence of aluminium humates, probably due to a decrease in the negative charge of the humic acid molecules after complexing The use of electroanalytical techniques for metal speciation studies in solls and natural water, if humic materials are known to be present, might therefore be limited
\end{abstract}

\section{INTRODUCTION}

The application of polarographic and voltammetric techniques to the study of metal speciation in natural and waste waters has received much attention [1-5]. With these methods, one can in principle distinguish between the different physico-chemical forms of metals that might be present in aqueous media. This approach could also be useful in studying metal humate reactions in soil solutions. However, systems involving natural organic substances are likely to be complex.

The possibility that some metals may form partially electroreducible complexes with these organic substances has been considered only recently [6-8]. Another problem which arises is the possible adsorption of organic materials on to the electrode surface [9]. This could lead to erroneously small values for "free" metal concentrations by decreasing the surface area available for electrode reactions and possibly restricting diffusion to the electrode. Conversely, electroreduction of metal-humate complexes, in solution and/or adsorbed on the electrode surface, at potentials similar to that of the "free" metal reduction 
can lead to overestimates of the uncomplexed metal concentration. As a result, before electroanalytical techniques may be used confidently in metal speciation studies involving humic acids, the possibility of adsorption must be investigated.

The potential of zero charge (pzc) is a fundamental characteristic of the interface between an electrode and its surrounding medium. The shift of the pzc with electrolyte concentration or the addition of an organic species to the solution (the Esin-Markov effect) is one measure of specific adsorption [10]. The extent and direction of the shift in the pzc provides useful information about the nature of the adsorbed substances.

Research into the effect of adsorbed organic species on the pzc of electrodes has been reviewed by several workers [11-13]. In general, specific adsorption of anions causes a shift of the pzc towards negative potential, and the opposite effect in the case of cations [11]. When both anions and cations are adsorbed, the net shift may be rather small because of mutual compensation, but may be detected by a flattening of the electrocapilary curve in the region of the maximum. Uncharged organic species tend to be adsorbed over a limited potential * range in the vicinity of the pzc. The presence of polar groups in the adsorbed molecule can influence the extent of the shift [12]. Strong $\pi$-bond interactions or the existence of permanent dipoles in the inner part of the double layer can cause much greater shifts in the pzc [12].

Several theories have been developed to describe the adsorption of flexible macromolecules $[14,15]$. Humic acids and their aluminium complexes are far more complicated then most of the polymers treated in these theories. At a surface-solution interface, humic acids cannot necessarily be expected to behave in a similar manner to well-characterised synthetic polymers and colloids. The adsorption of the latter has been found to be complicated by multilayer formation [16], and both flocculation or changes in configuration (e.g. uncoiling) upon entering the double layer. Consequently, careful consideration must be made of the physicochemical properties of the humic acid species present in solution before any results may be interpreted.

The colloidal and polyelectrolytic behaviour of humic acids has been reviewed by Hayes and Swift [17]. From the experimental evidence available, it would appear that the humic acid molecules may be considered to be a flexible random coll. The molecules are essentially spherical in shape, and within these spheres, the radial distribution of mass and charge may be described by a Gaussian function. This picture of the shape of the molecules is also supported by their swelling properties and ion-exchange behaviour [17]. The latter indicate that the molecules are readily penetrated by solvent and that most charges sites are associated and available for ion exchange. It has been shown [18] that neither variation in $\mathrm{pH}$ nor complexing with aluminum ions changes the shape of the molecules significantly, but it does alter the radial distribution of the mass, i.e. as the $\mathrm{pH}$ is decreased, or complexing with polyvalent cations increases, the Gaussian function describing the mass distribution narrows. There is no evidence that humic acids can behave as zwitterions [17]. The possibility cannot be ruled out that when it is complexed with polyvalent metal ions, the molecule can attain a slight positive charge. This charge would result from complete saturation of the negative sites on the humic acid molecule 
leaving an excess of positive charge on some of the attached polyvalent cations. Even so, the amount of positively charged species will be very small, because such molecules have been found to be very unstable in solution and precipitate before any appreciable amount of positive charge can build up [17].

In this paper, the pzc of mercury in the presence of soil humic acids and their aluminium complexes is measured using the drop-time method. This technique has been used by many workers [19-22]. Experimental errors can arise from variations in the flow rate of the mercury due to back pressure and from the shape of the capillary [23,24]. However, it has been calculated [25] that the errors arising from the former source are $<2 \%$ and providing a long column of mercury and the same capllary are used for all measurements, the effect is insignificant [19]. The latter effect is minimised by using tapered capillaries to ensure that as many parts of the surface of the drop as possible are at the same equilibrium with the surrounding solution.

\section{EXPERIMENTAL}

\section{Reagents and glassware}

Analytical grade chemicals were used except where noted, and the water was distilled and deionised. All glassware was cleaned by ultrasonification in $1: 1$ nitric acid for a few minutes. The mercury was washed in nitric acid and then twice distilled as described by Vogel [26].

\section{Aluminium stoch solutions}

Aluminium (99.999\% Koch-Light) was dissolved in a slight excess of purestgrade 20\% hydrochloric acid (Hayashı Pure Chemical Industries) and the solution was diluted to a concentration of $3.71 \times 10^{-2}$ mol dm ${ }^{-3}$ with $1.5 \times 10^{-2}$ mol $\mathrm{dm}^{-3}$ nitric acid. A dulute stock solution was then prepared by diluting $3.37 \mathrm{~cm}^{3}$ of the above to $100 \mathrm{~cm}^{3}$ with $1.5 \times 10^{-2}$ mol dm $\mathrm{dm}^{-3}$ nitric acid.

\section{Preparation of humic acid stock solutions and samples}

Humic acids were extracted with $5 \times 10^{-1} \mathrm{~mol} \mathrm{dm}^{-3}$ sodium hydroxide from a highly humified, organic muck soil as described previously $[27,28]$; this method produced samples low in organic and inorganic impurities. The humic acids had an average molecular weight of approximately 60,000 and an average diffusion coefficient in $5 \times 10^{-2} \mathrm{~mol} \mathrm{dm}^{-3}$ potassium nitrate solution of $6 \times$ $10^{-7} \mathrm{~cm}^{2} \mathrm{~s}^{-1}[18]$.

A $0.015 \%$ solution was prepared by dissolving the freeze-dried humic acid in $5 \times 10^{-2} \mathrm{~mol} \mathrm{dm}^{-3}$ potassium carbonate, and passing the solution through a hydrogen-saturated resin until there was no further change in $\mathrm{pH}$. The broadening of a narrow beam of light passed through this solution (whicls had a final $\mathrm{pH}$ of $\mathrm{ca}$. 3.0) indicated that the humic acids were collordal in nature. Aliquots of this suspension were then passed through a sodium-saturated resin to prepare five stock solutions of humic acids at $\mathrm{pH}$ values of $3.5,4.0,5.0,7.0$ and 9.0. The humic acids were totally dispersed in solution at $\mathrm{pH} \geqslant 7.0$ as there 
was no Tyndall beam effect in these preparations. All solutions were deaerated with high-purity nitrogen (Commonwealth Industrial Gases) to remove carbon dioxide before $\mathrm{pH}$ measurements were made.

Humic acid samples were prepared by adding $1.33 \mathrm{~cm}^{3}$ of a $1.125 \mathrm{~mol} \mathrm{dm} \mathrm{dm}^{-3}$ potassium nitrate solution to $0-28 \mathrm{~cm}^{3}$ of each humic acid stock solution and diluting to $30 \mathrm{~cm}^{3}$. This gave an ionic strength of $5 \times 10^{-2} \mathrm{~mol} \mathrm{dm}^{-3}$. The $\mathrm{pH}$ of each sample was then adjusted back to that of the original stock solution using an automatic titration assembly (Radiometer) in the $\mathrm{pH}$ stat mode. The addition of acid or alkali did not alter the ionic strength by more than $1 \%$.

\section{Preparation of aluminum humates}

In order to be able to prepare aluminium humate complexes with various humic acid : aluminium ratios, the cation exchange capacity (cec) of humic acids was used as an estimate of the number of sites that could be involved in binding cations. This quantity was determined for each $\mathrm{pH}$ using the cec value of 297 milliequivalents per $100 \mathrm{~g}$ at $\mathrm{pH} 7.0$, calculated from titration curves, and the variation of the degree of dissociation with $\mathrm{pH}$ estimated by Posner [29]. The amount of aluminium required to give the various humic acid : aluminium ratios could then be estimated and a series of samples were prepared in a similar fashion to that described above.

\section{Apparatus}

A silver -silver chloride electrode (dipping in saturated potassium chloride solution) and a platinum auxiliary electrode were used in conjunction with a potentiostat (P.A.R. model 173) to control the potential of a dropping mercury electrode (DME) and to measure the current flowing through the drop. The DME had a tapered capillary which had been siliconized.

\section{Procedure}

Each sample was deaerated with high-purity nitrogen for at least $10 \mathrm{~min}$ if the $\mathrm{pH}$ was $<7.0$ and for $2 \mathrm{~h}$ if the $\mathrm{pH}$ was $\geqslant 7.0$. The DME was then introduced into the sample vessel and the nitrogen redirected over the surface of the solution. The height of the mercury reservoir above the sample was kept constant at a level such that freely falling drops had a lifetime of about 5-6 s. The time for $c a$ 60-70 drops to fall at a preselected potential was measured using a stop-watch accurate to $0.01 \mathrm{~s}$. Concurrently, the change in current during drop life was plotted on an $\mathrm{x}-\mathrm{t}$ recorder; each deflection represented one drop and these were counted to give the actual number of drops that had fallen during the measured time. The procedure was repeated, over a range of potentials in the vicinity of the pzc, at $50 \mathrm{mV}$ intervals in order to construct a graph of time of drop life vs. potential. A curve was fitted through the points by polynomial regression analysis and the potential at the maximum (i.e. the pzc) was estimated from the polynomial. The error in this type of calculation is $\pm 5 \mathrm{mV}$. Results are reported relative to the silver/silver chloride electrode. 


\section{Equlibration time}

The most commonly used experimental methods for determining the pzc of mercury are based on measurements made at either a stationary (hanging mercury drop) or a moving (dropping mercury) electrode [12]. It has been observed [30] that when a stationary electrode is used, equilibration times of the order of $15 \mathrm{~min}$ may be required before accurate measurements can be made. This phenomenon is probably due to slow adsorption processes and/or slow movement of the species to the electrode surface. Parsons [31] has shown that when using a moving electrode, equilibration time is significantly shortened by natural convection which speeds up diffusion to the drop.

In order to determine whether adsorption equilibrium is likely to be attained by the drop lifetime in our measurements, estimates of the pzc of a humic acid solution were made using mercury drop with approximate lifetimes of $5,7,10$ and $11 \mathrm{~s}$. The results in Table 1 indicate that the time required for the adsorption of humic acids to achieve equilibrium is $\leqslant 5 \mathrm{~s}$. Several workers $[30,32,33]$ have found that extensive rearrangements occurring during the adsorption process result in times $>\mathbf{3 0} \mathrm{s}$ being required before equilıbrium is achieved. In comparison, the relatively short time required for equilibrium of humic acid adsorption suggests a minimum of distortion from the mean configuration in the bulk solution. This would be the most favoured rearrangement, if any, since such a process would be accompanied by the least change in free energy. However, the energy changes involved in rearrangement would have to be balanced against the free energy changes derived from concentration changes and solvent-surface interactions.

\section{Drop time us. potential curves}

As expected, the curve of drop time vs. potential was approximately parabolic in shape, some typical results being given in Fig. 1.

There was no significant difference between the measurements of the pzc of mercury in $5 \times 10^{-2} \mathrm{~mol} \mathrm{dm}^{-3}$ potassium nitrate, the background electrolyte, at different $\mathrm{pH}$ values. The mean value was found to be $-470 \mathrm{mV}$ with a coefficient of variation of $\mathbf{2 . 5 \%}$. This was in agreement with values found by Payne [34].

\section{TABLE 1}

The pzc of mercury in the presence of $833 \times 10^{-6} \mathrm{~mol} \mathrm{dm}^{-3}$ humic acid at $\mathrm{pH} 40$, measured as a function of drop time

\begin{tabular}{ll}
\hline Drop time/s & pzc $E_{\sigma=0} / \mathrm{mV}$ \\
\hline 5 & 546 \\
7 & 546 \\
10 & 549 \\
11 & 544 \\
\hline
\end{tabular}




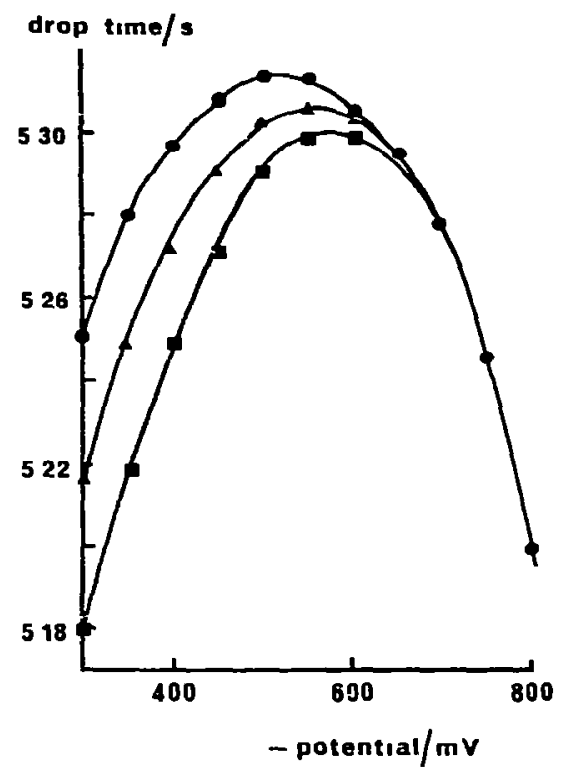

Fig. 1 Variation in the drop lifetime with potentıal for $8.33 \times 10^{-6} \mathrm{~mol} \mathrm{dm}^{-3}$ humic acids at different $\mathrm{pH}$ values (•) $\mathrm{pH} 35,(\Delta) \mathrm{pH} 4.0$, (E) $\mathrm{pH} 5.0$

\section{TABLE 2}

The pzc of mercury in the presence of humic acids and their complexes with aluminium $(-\mathrm{mV})^{\alpha}$

\begin{tabular}{|c|c|c|c|c|c|c|}
\hline \multirow[t]{2}{*}{ pH } & \multirow{2}{*}{$\begin{array}{l}\text { Humic acid } \\
\text { Al ratio }\end{array}$} & \multicolumn{5}{|c|}{ Humic acid concentration $10^{6} / \mathrm{mol} \mathrm{dm}^{-3}$} \\
\hline & & 136 & 2.75 & 4.17 & 5.58 & 833 \\
\hline 35 & $\begin{array}{ll}1 & 0 \\
1 & 1\end{array}$ & $\begin{array}{l}480 \\
470\end{array}$ & $\begin{array}{l}485 \\
475\end{array}$ & $\begin{array}{l}490 \\
485\end{array}$ & $\begin{array}{c}505 \\
-\end{array}$ & $\begin{array}{l}515 \\
490\end{array}$ \\
\hline 4.0 & $\begin{array}{ll}1 & 0 \\
4 & 1 \\
2 & 1 \\
3 & 2 \\
1 & 1\end{array}$ & $\begin{array}{l}475 \\
475 \\
475 \\
455 \\
470\end{array}$ & $\begin{array}{l}485 \\
485 \\
485 \\
480 \\
500\end{array}$ & $\begin{array}{l}510 \\
495 \\
490 \\
490 \\
480\end{array}$ & $\begin{array}{c}540 \\
- \\
- \\
-\end{array}$ & $\begin{array}{l}\mathbf{5 4 5} \\
\mathbf{5 3 5} \\
\mathbf{5 3 0} \\
\mathbf{5 2 0} \\
\mathbf{5 1 5}\end{array}$ \\
\hline 50 & $\begin{array}{lr}1 & -0 \\
1 & 5 \cdot 1\end{array}$ & $\begin{array}{l}475 \\
465\end{array}$ & $\begin{array}{l}490 \\
465\end{array}$ & $\begin{array}{l}535 \\
485\end{array}$ & 560 & $\begin{array}{l}580 \\
530\end{array}$ \\
\hline 70 & $\begin{array}{ll}1 & 0 \\
18 & 1\end{array}$ & $\begin{array}{l}490 \\
490\end{array}$ & $\begin{array}{l}525 \\
505\end{array}$ & $\begin{array}{l}540 \\
525\end{array}$ & $\begin{array}{c}565 \\
-\end{array}$ & $\begin{array}{l}580 \\
555\end{array}$ \\
\hline 90 & $\begin{array}{lll}1 & & 0 \\
1 & 2 & 1\end{array}$ & 480 & $\begin{array}{l}520 \\
520\end{array}$ & 535 & $\stackrel{555}{-}$ & $\stackrel{590}{-}$ \\
\hline
\end{tabular}

a The pzc of mercury in the supporting electrolyte, $5 \times 10^{-2} \mathrm{~mol} \mathrm{dm} \mathrm{m}^{-3}$ potassium nitrate, was $-470 \mathrm{mV}$ 
As can be seen from Fig. 1, in the presence of humic acid (or its complexes with aluminium), there was a net overall shift in the pzc of the mercury towards negative potentials, a complete set of results being given in Table 2 . The negative direction of the shift suggests that the molecular dipoles are oriented with the negative end towards the surface [35]. The greatest shift was observed when mercury was in the presence of the highest concentration of humic acid at the highest $\mathrm{pH}$. This shift to more negative potentials is indicative of the presence of aromatic neutral and/or negatively charged molecules [12], both of which are almost certainly present in a solution of humic acid. Over the range of experimental conditions studied, the shift was not very great (i.e. $<150 \mathrm{mV}$ ). If uncoiling of the molecule was occurring during adsorption, one might expect a very large shift in the pzc as more charged sites came into contact with the surface. The size of the shift would then be more comparable with that observed for the adsorption of linear polymers [36].

Even though the drop times decreased with specific adsorption, there was no significant change in the shape of the curves. This is further evidence that uncoiling was not occurring since it has been shown that this sort of rearrangement results in a flattening of the electrocapillary curve [32].

\section{Variation in the pzc with $p H$ and humic acid concentration}

In the presence of a constant concentration of humic acid, the shift in pzc of the mercury increased sigmoidally with increasing $\mathrm{pH}$ (Fig. 2).

In discussing the behaviour of humic acid as a weak polyacid, one must take into account that the ionisable groups which are present are probably interdependent, and this interdependence may vary with changes in the environment (e.g. pH). Consequently, when considering the dissociation of humic acid (and its possible metal-binding reactions), it must be noted that it is a polymeric, weak polyelectrolyte and its behaviour tends to reflect a net response of

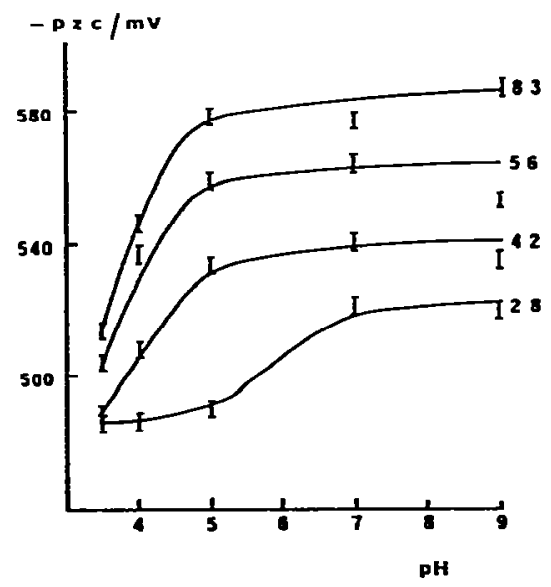

Fig. 2. Variation in the pze of mercury with $\mathrm{pH}$ in the presence of different humic acid concentrations. The numbers represent $10^{6} \times$ humic acid concentration in $\mathrm{mol} \mathrm{dm}^{-3}$. 
each molecule separately and/or in combination. The $\mathrm{p} K$ values are, therefore, mean estimates of a Gaussian distribution of acid $p K$ values which will vary within as well as between molecules. As the $\mathrm{pH}$ is increased, more humic acid will dissociate to replace those $\mathrm{H}^{+}$ions neutralised by the increased $\mathrm{OH}^{-}$concentration, and consequently the polarity of the molecule will increase. Therefore, at constant concentration and varying $\mathrm{pH}$ the shift in pzc could be associated with the adsorption of the ionised species whose concentration in solution increases as the $\mathrm{pH}$ rises. The increase in the shift with increasing $\mathrm{pH}$ indicates an increase in the component of the molecular dipole perpendicular to the surface [35]. This could be attributed to the charged species present which may not necessarily be preferentially adsorbed, but represent a greater proportion of the humic acid concentration at the higher $\mathrm{pH}$ values.

An estimate of the average $\mathrm{pK}$ of the humic acid can be made by noting the $\mathrm{pH}$ at which the rate of change of the pzc is greatest [30]. This value of the $\mathrm{pK}$ describes the acid-base equilibrium at the surface which need not be the same as that of the bulk solution. Doljido et al. [30] derived an approximate relationship between the $\mathrm{p} K$ at the surface $\left(\mathrm{p} K_{\mathrm{s}}\right)$ and that in the bulk solution $\left(\mathrm{p} K_{\mathrm{b}}\right)$ :

$\mathrm{p} K_{\mathrm{s}}=\mathrm{p} K_{\mathrm{b}}+\log (\beta / \bar{\beta})$

where $\beta$ and $\bar{\beta}$ are the adsorption constants for change and uncharged species respectively. The relationship assumes low values of $\beta$, small concentrations of the adsorbed species and that their surface concentrations would be equal when either fully covered that surface. These assumptions are reasonable in this particular case because the adsorption of large molecules at relatively low concentrations is being studied.

The $p K$ value calculated for similar humic acids by Posner [29] is 4.5, whereas that determined from pzc data is approximately 4.25 , except at the

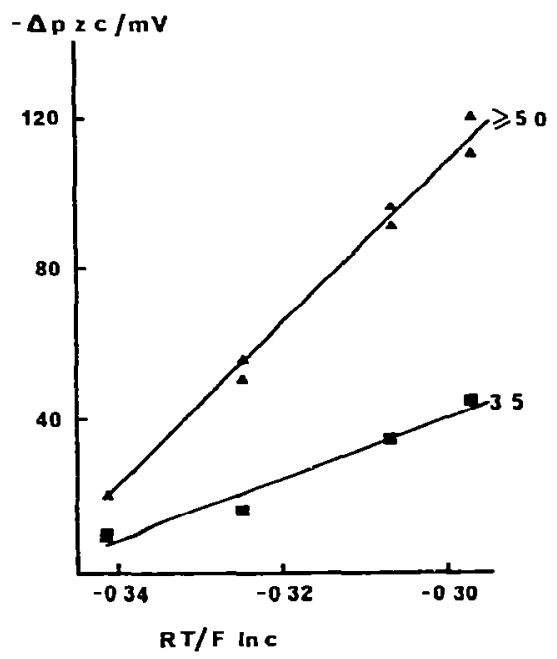

Fig 3. Variation in the shift of pzc ( $\Delta$ pzc) of mercury with $(R T / F)$ in $c$ for humic acids at different $\mathrm{pH}$ values $(c=$ concentration). 
lowest concentration studied. In the latter case, the $\mathrm{p} K$ value is approximately 6.0, indicating that adsorption of uncharged species is greater than that of charges species. When the pH of the solution was kept constant and the humic acid concentration was increased, the pzc also shifted cathodically. The curves in Fig. 3 are similar to Esin-Markov plots [10], They were found to be approximately linear over the concentration range studied. The slope increased with increasing $\mathrm{pH}$, but reached a limiting value at $\mathbf{p H} 5$.

In this system, as the concentration increases, the amounts of charged and uncharged species will increase approximately in the same proportion. Adsorption of uncharged molecules and some $\pi$-bond interaction are evidenced by the shift in pzc of mercury in humic acid at pH 3.5, where there is nearly complete association. These molecules may be in the colloidal state and/or may have disaggregated upon adsorption.

\section{Variation in the pzc with $\mathrm{pH}$ and aluminium humate concentration}

An approximately linear relationship was found between the pzc and the concentration of metal humate complex, with the slope of the line increasing with increasing pH (Fig. 4). This suggests a variation in the proportion of adsorption attributable to the more negative species.

At pH 3.5, and a humic acid : aluminium ratio of almost unity which was very close to the point of precipitation, the solution could be considered to consist primarily of neutral or positively charged species. However, as men-. tioned above, positively charged molecules will form a very minor proportion of the species in solution. The low angle of slope of the variation of pzc with concentration is consistent with this hypothesis. At pH 7.0, where the humic acid : aluminium ratio was less than unity, not all the ionised species had been reacted with aluminium and consequently, the slope of the line is much greater.

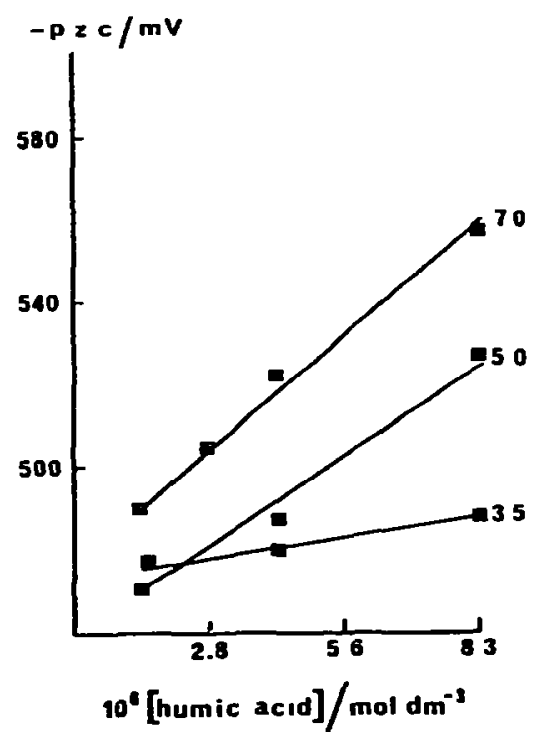

Fig. 4. Variation in the pzc of mercury with concentration and $\mathrm{pH}$ of aluminium humate complexes 
When aluminium was complexed with humic acid in varying ratios, it was found that the shift in pzc to more negative values increased with decreasing proportion of aluminium complexed. This may be explained by complexing of previously ionised functional groups to give chelates whose adsorption has a lesser effect on the pzc of mercury by virtue of their decrease in net charge upon complexing. The possibility of the presence of positively charged aluminium humate molecules must be considered for complexes where the humic acid is saturated with aluminium and very near its point of precipitation. Adsorption of neutral or slightly positively charged molecules could lead to a flattening of the electrocapillary curve or to a small positive shift in the pzc which was not observed. The observed changes in pzc are probably a net result of the adsorption of several different, less negatively charged species, in equilibrium with each other.

\section{CONCLUSION}

The results indicated that humic acids and their complexes with aluminium are specifically adsorbed on to mercury. Consequently, the application of electroanalytical techniques to the study of metal-humic acid reactions in the soil or natural waters is somewhat limited until more quantitative information is available concerning the extent and effects of such adsorption.

\section{ACKNOWLEDGEMENT}

One of us (G.S.P.R.) gratefully acknowledges the award of a University of Western Australia Research Scholarship.

\section{REFERENCES}

1 K H Mancy in S.F. Jenkins (Ed), Progress in Water Technology, Vol 3, Pergamon Press, New York, 1973. p 63.

2 R D. Guy and C L Chakrabarti, Proc Int. Conf. on Heavy Metals in the Envronment, Toronto, Canada, 1975, p 275.

3 T.M Florence and G E. Batley. Talanta, 24 (1977) 151

4 W. Davson and $M$ Whitfield. J. Electroanal Chem, 75 (1977) 763

5 H P van Leeuwen, J. Electroanal. Chem , 99 (1978) 93.

6 P.L. Brezonuk. P A Brauner and W. Stumm, Water Res., 10 (1976) 605

7 H P van Leeuwen. Anal. Chem.. 61 (1979) 1322.

8 W. Danson. J. Electroanal. Chem., 87 (1978) 395.

9 J. Buffe, A Commol, F.L. Greter and W. Haerdı, Proc. Anal Div. Chem. Soc., 1978, p. 59

10 P. Delahay, Double Layer and Electrode Kinetics, Interscience, New York, 1965, p. 53.

11 I.M Kolthoff and J J Lingane. Polarography, Vol 1, Interscience. New York, 1952.

12 S.D. Argade and E. Guleadi in E. Gleadi (Ed). Electrosorption, Plenum Press, New York, 1967, p. 87.

13 B B Damaskın. O A. Petri and V.V. Batrakov, Adsorption of Organic Compounds on Electrodes. Plenum Press, New York, 1971.

14 A Suberberg, J. Chem. Phys., 48 (1968) 2835.

15 R J. Roe, J. Chem. Phys., 60 (1974) 4192.

16 R.G. Barradas and F.M Kimmerlen, J. Electroanal. Chem, 9 (1965) 483.

17 M H B. Hayes and R S. Swift in D J. Greenland and M H B Hayes (Eds ), The Chemistry of Soll Constituents, Wley, Chichester, UK, 1978, p. 179.

18 G. Ritchie, unpublished results. 
19 R. Parsons, Rev. Pure Appl. Chem., 18 (1968) 91.

20 S. Trasatt.. J. Electroanal. Chem., 28 (1970) 257.

21 A. Mazhar, R. Bennes, P. Vanel and D. Schuhmann, J. Electroanal. Chem., 100 (1979) 395.

22 J. Dittrich and E. Kretschmer, J. Electroanal. Chem., 107 (1980) 391.

23 D.C. Grahame, J. Am. Chem. Soc., 68 (1946) 301.

24 R Parsons and P.C. Symons, Trans. Faraday Soc., 64 (1968) 1077.

25 D.C. Grahame, J. Am. Chem. Soc.. 79 (1957) 2093.

26 A.I. Vogel, A Textbook of Quantitative Inorganic Analysis, Longman. London, 1969.

27 A.M. Posner, J. Soll. Sci . 17 (1966) 65.

28 A M. Posner, B K.G. Theng and J.R.H. Wake, Trans. 9th Int. Congr. Soll Sel , 3 (1966) 153.

29 A.M. Posner, Trans. 8th Int. Congr. Soil. Sc1., 3 (1964) 161.

30 J. Dojlddo, M. Dmowska-Stanczak and Z. Galus, J. Electroanal Chem., 94 (1978) 107

31 R. Parsons, Adv. Electrochem. Electrochem. Eng., 1 (1961) 27.

32 I R. Miller, Trans Faraday Soc, 57 (1961) 301.

33 W.H. Grant. L.E. Smith and R.R. Stromberg. Faraday Discuss. Chem. Soc., 59 (1975) 209

34 R. Payne, J. Electrochem. Soc., 113 (1966) 999.

35 S. Trasattı, J. Electroanal. Chem , 53 (1974) 335

36 J.K. Koopal and J. Lyklema, Faraday Discuss, Chem. Soc., 59 (1975) 230. 